\title{
HEEGAARD FLOER GENUS BOUNDS FOR DEHN SURGERIES ON KNOTS
}

\author{
STANISLAV JABUKA
}

\begin{abstract}
We provide a new obstruction for a rational homology 3-sphere to arise by Dehn surgery on a given knot in the 3 -sphere. The obstruction takes the form of an inequality involving the genus of the knot, the surgery coefficient, and a count of $L$-structures on the 3 -manifold, that is $\operatorname{spin}^{c}$-structures with the simplest possible associated Heegaard Floer group. Applications include an obstruction for two framed knots to yield the same 3-manifold, an obstruction that is particularly effective when working with families of framed knots. We introduce the rational and integral Dehn surgery genera for a rational homology 3 -sphere, and use our inequality to provide bounds, and in some cases exact values, for these genera. We also demonstrate that the difference between the integral and rational Dehn surgery genera can be arbitrarily large.
\end{abstract}

\section{INTRODUCTION}

1.1. Preface. It is well known [6, 21] that every oriented, closed 3-manifold can be constructed via Dehn surgery on a framed link $L$ in $S^{3}$. The framed link $L$ in this construction is highly non-unique, but any two framed links yielding the same 3-manifold are related by a finite sequence of blow-ups, blow-downs, handle slides and isotopies [5] (two such framed links shall be called surgery equivalent). While in theory this curbs the non-uniqueness, in practice it is often not easy to tell if two framed links are related in this manner. Indeed, even in the simpler case of framed knots, it remains a challenge. The first example of an integral homology sphere that can be obtained by surgeries on two different knots was found by Lickorish [7, and many examples have followed since then [1, 4, 8, 19, 20].

To help restrain this many-to-one phenomenon, we derive an obstruction for a 3manifold $Y$ to be the result of a $p / q$-framed surgery on a knot $K \subset S^{3}$. The obstruction takes the form of an inequality (Theorem 1.3) involving $p, q$, the genus of the knot $K$ and data derived from the Heegaard Floer homology groups of $Y$.

For a given framed knot, this inequality bounds from below the genus of any surgery equivalent framed knot.

Among framed links in $S^{3}$, those with integer framings play a special role. Indeed, any such link $L$ does not only yield 3-manifold $Y$ via Dehn surgery, but also describes a smooth, oriented 4-manifold $X$ with $\partial X=Y$, obtained by attaching 4-dimensional 2-handles to the 4-ball $D^{4}$, attached to the link $L \subset \partial D^{4}$. For this reason, we shall heed special attention to integral surgeries when stating our results.

The author was partially supported by grant \#246123 from the Simons Foundation, and from a research grant from the University of Nevada, Reno. 
1.2. Definitions. If $r=p / q$ is a rational number in lowest terms, we shall write $S_{r}^{3}(K)$ or $S_{p / q}^{3}(K)$ to denote the 3-manifold resulting from $r$-framed Dehn surgery on the knot $K \subset S^{3}$. The Seifert genus of a knot $K$ shall be denoted $g(K)$.

Definition 1.1. Let $Y$ be a rational homology 3-sphere. We define its rational and integral Dehn surgery genera $g_{\mathbb{Q}}(Y)$ and $g_{\mathbb{Z}}(Y)$ as:

$$
\begin{gathered}
g_{\mathbb{Q}}(Y)=\left\{\begin{array}{cll}
\min \left\{g(K) \mid Y=S_{r}^{3}(K), r \in \mathbb{Q} .\right\} & ; & \text { If } Y=S_{r}^{3}(K) \text { for some } K . \\
\infty & \text { Otherwise. }
\end{array}\right. \\
g_{\mathbb{Z}}(Y)=\left\{\begin{array}{cl}
\min \left\{g(K) \mid Y=S_{p}^{3}(K), p \in \mathbb{Z} .\right\} & \text { If } Y=S_{p}^{3}(K) \text { for some } K . \\
\infty & ; \quad \text { Otherwise. }
\end{array}\right.
\end{gathered}
$$

Note that $g_{\mathbb{Q}}(Y)=0$ if and only if $Y$ is a lens space.

For a closed and oriented 3-manifold $Y$, let $\operatorname{Spin}^{c}(Y)$ denote its affine space of spin $^{c}$-structures and let $\widehat{H F}(Y, \mathfrak{s})$ be its associated hat version of the Heegaard Floer homology group (these are defined in Section 2.2. .

Definition 1.2. Let $Y$ be a rational homology 3-sphere. A $\operatorname{spin}^{c}$-structure $\mathfrak{s} \in \operatorname{Spin}^{c}(Y)$ is called an $L$-structure if $\widehat{H F}(Y, \mathfrak{s}) \cong \mathbb{Z}$. We shall write $\ell(Y)$ or simply $\ell$ to denote the number of $L$-structures on $Y$.

Our use of nomenclature follows that of [13] where a rational homology sphere, all of whose $\operatorname{spin}^{c}$-structures are $L$-structures, is called an $L$-space.

1.3. Results. With these definitions in place, we turn to our surgery obstruction.

Theorem 1.3. Let $Y$ be a rational homology sphere with $\left|H_{1}(Y ; \mathbb{Z})\right|=p$. If $Y$ is obtained by $p / q$-surgery on a knot $K \subset S^{3}$ with $g(K) \geq 1$, then

$$
2 g(K)-1 \geq \frac{p-\ell}{|q|} .
$$

Here $\ell$ is the number of L-structures on $Y$.

Using different approaches, other genus bounds stemming from Heegaard Floer homology for knots with prescribed surgeries have been obtained by Ozsáth and Szabó [13] (providing four-ball genus bounds for knots with lens space surgeries), Rasmussen [15] (showing that if a surgery of slope $p$ on a genus $g$ knot yields a lens space, then $p \leq 4 g+3$ ) and Greene [3] (demonstrating the inequality $2 g-1 \leq p-\sqrt{3 p+1}$ for a knot $K$ of genus $g$ on which integral $p>0$ surgery yields a lens space that bounds a sharp 4-manifold with torsion-free first homology).

Corollary 1.4. Let $Y$ be a rational homology sphere different from a lens space, and let $\ell$ be the number of $L$-structures on $Y$. Then

$$
2 g_{\mathbb{Z}}(Y)-1 \geq\left|H_{1}(Y ; \mathbb{Z})\right|-\ell .
$$

Remark 1.5. Inequality (1) from Theorem 1.3 unfortunately becomes vacuous for $L$ spaces and integral homology spheres. In both cases the inequality reduces to $g(K) \geq 1$ which is a hypothesis of the theorem. 
1.4. Examples. We provide families of examples to illustrate two points:

(a) Inequality (1) from Theorem 1.3 is sharp for infinitely many surgeries (Proposition 1.6 and Example 1.7).

(b) Theorem 1.3 can be used to provide infinitely many examples of 3-manifolds $Y$ for which $g_{\mathbb{Z}}(Y)>g_{\mathbb{Q}}(Y)$. Indeed, the difference $g_{\mathbb{Q}}(Y)-g_{\mathbb{Z}}(Y)$ can be made arbitrarily large, while being finite (Example 1.8).

For a knot $K$ in $S^{3}$, let $\tau(K)$ denote its Ozsváth-Szabó tau invariant [9] (see Section 2.5 for a detailed definition).

Proposition 1.6. Let $K \subset S^{3}$ be a knot with $|\tau(K)|=g(K)>0$ and let $p, q$ be a pair of positive, relatively prime integers with $p-(2 g(K)-1) q>0$. Then

$$
\ell\left(S_{\varepsilon \frac{p}{q}}^{3}(K)\right)=p-(2 g(K)-1) q
$$

where $\varepsilon=-\operatorname{Sign}(\tau(K))$.

Any knot $K$ as in Proposition 1.6 renders inequality (1) sharp. Explicit examples of such knots are provided by $L$-knots (knots which yield an $L$-space by some positive, integral surgery [13], for instance torus knots $T_{(a, b)}$ with $a b>0$ ) and their mirrors, and alternating knots $K$ with signature $\sigma(K)= \pm 2 g(K)$.

Example 1.7. Let $K$ be a knot meeting the hypothesis of Proposition 1.6 and set $\varepsilon=-\operatorname{Sign}(\tau(K))$. Then, for any positive integer $p>2 g(K)-1$, one obtains

$$
g_{\mathbb{Z}}\left(S_{\varepsilon p}^{3}(K)\right)=g(K) .
$$

For instance, taking a positive integer $g$ and letting $K$ be the torus knot $T_{(2,2 g+1)}$, one obtains $g_{\mathbb{Z}}\left(S_{-p}^{3}\left(T_{(2,2 g+1)}\right)\right)=g($ still with $p>2 g-1)$.

Computations justifying our claims in the next example are deferred to Section 4 .

Example 1.8. We exhibit an infinite family of rational homology 3-spheres $Y_{n}$ for which $g_{\mathbb{Z}}\left(Y_{n}\right)-g_{\mathbb{Q}}\left(Y_{n}\right) \geq \frac{n-1}{2}$. Namely, for $n \in \mathbb{N}$ let $Y_{n}$ be the result of $-\frac{4 n+1}{n}$-framed surgery on the Figure Eight knot. Then $\ell\left(Y_{n}\right)=3 n+1$ so that $Y_{n}$ is not an $L$-space for any choice of $n$. Since the genus of the Figure Eight knot is 1 , it follows that $g_{\mathbb{Q}}\left(Y_{n}\right)=1$. Inequality (2) shows that $g_{\mathbb{Z}}\left(Y_{n}\right) \geq \frac{n+1}{2}$ leading to $g_{\mathbb{Z}}\left(Y_{n}\right)-g_{\mathbb{Q}}\left(Y_{n}\right) \geq \frac{n-1}{2}$. In Section 4 we show that $Y_{n}$ also arises as an integral surgery on a knot showing $g_{\mathbb{Z}}\left(Y_{n}\right)-g_{\mathbb{Q}}\left(Y_{n}\right)$ to be finite.

1.5. Applications. As already alluded to in the introduction, Theorem 1.3 can obstruct surgery equivalence among framed knots. We remark that we are only using the ranks of the Heegaard Floer groups for this obstruction. In another direction, the Heegaard Floer correction terms can also be used to furnish surgery obstructions, see for instance [2].

For a pair of framed knots $\left(K_{1}, \frac{p}{q_{1}}\right)$ and $\left(K_{2}, \frac{p}{q_{2}}\right)$, the obstruction is evaluated by computing the number $\ell$ of $L$-structures on $Y=S_{p / q_{1}}^{3}\left(K_{1}\right)$, and by asking whether the inequality

$$
2 g\left(K_{2}\right)-1 \geq \frac{|p|-\ell}{\left|q_{2}\right|}
$$


is violated. If the answer is 'Yes', then $\left(K_{1}, \frac{p}{q_{1}}\right)$ and $\left(K_{2}, \frac{p}{q_{2}}\right)$ are not surgery equivalent.

A comparison of the Heegaard Floer homology groups for $S_{p / q_{1}}^{3}\left(K_{1}\right), S_{p / q_{2}}^{3}\left(K_{2}\right)$ is of course a stronger obstruction to surgery equivalence, but it also involves more computation. This becomes especially prominent when $K_{2}$ is not fixed but allowed to vary across a family of knots. In such a happenstance, Theorem 1.3 can be used as a significant shortcut to ruling out surgery equivalence. We illustrate this point with two examples.

Example 1.9. Consider a pair of surgery equivalent framed knots $\left(K_{1},-\frac{p}{q_{1}}\right)$ and $\left(K_{2},-\frac{p}{q_{2}}\right)$ with $p, q_{i}>0, \operatorname{gcd}\left(p, q_{i}\right)=1$ and $p-\left(2 g\left(K_{i}\right)-1\right) q_{i}>0$.

(i) If $\tau\left(K_{1}\right)=g\left(K_{1}\right)$ then

$$
2 g\left(K_{2}\right)-1 \geq \frac{q_{1}}{q_{2}} \cdot\left(2 g\left(K_{1}\right)-1\right) .
$$

(ii) If $\tau\left(K_{i}\right)=g\left(K_{i}\right)$ for $i=1,2$

$$
2 g\left(K_{2}\right)-1=\frac{q_{1}}{q_{2}} \cdot\left(2 g\left(K_{1}\right)-1\right) .
$$

We are fixing the knot $K_{1}$ and allowing $K_{2}$ to vary through the family of all knots in $S^{3}$ (in part (i)) or through the family of knots with $\tau\left(K_{2}\right)=g\left(K_{2}\right)$ (in part (ii)). In each case, an application of Theorem 1.3 gives considerable restrictions on the genera and framings involved. For instance, if $K_{1}$ and $K_{2}$ in case (ii) above are of equal Seifert genus $g$, then the surgery equivalence of $\left(K_{1},-\frac{p}{q_{1}}\right)$ and $\left(K_{2},-\frac{p}{q_{2}}\right)$ implies that $q_{1}=q_{2}$.

Example 1.10. For positive integers $m, k$, let $K_{2 m, 2 k+1}$ be the knot in Figure 1. It is easy to check that $g\left(K_{2 m, 2 k+1}\right)=m$. In this example we apply Theorem 1.3 to give a partial answer to the question: When are the framed knots $\left(K_{2 m, 2 k+1}, \frac{p}{q_{1}}\right)$ and $\left(K_{2 n, 2 j+1}, \frac{p}{q_{2}}\right)$ surgery equivalent?

Assume that $p, q_{i}, p-(2 m-1) q_{1}, p-(2 n-1) q_{2}$ are all positive. We will show in Section 4 that

$$
\ell=\ell\left(S_{-p / q_{1}}^{3}\left(K_{2 m, 2 k+1}\right)\right)=p-m q_{1} .
$$

Theorem 1.3 gives the restriction

$$
\frac{q_{2}}{q_{1}} \geq \frac{m}{2 n-1}
$$

for any framed knot $\left(K_{2 n, 2 j+1},-\frac{p}{q_{2}}\right)$ surgery equivalent to $K\left(2 m, 2 k+1,-\frac{p}{q_{1}}\right)$.

How good an obstruction is this? In Section 4 we will demonstrate that with the choices of $p=4 m n-1, q_{1}=n, q_{2}=m$ and $j=k$, the framed $\operatorname{knot}\left(K_{2 m, 2 k+1},-\frac{4 m n-1}{n}\right)$ is surgery equivalent to $\left(K_{2 n, 2 k+1},-\frac{4 m n-1}{q_{2}}\right)$ with $q_{2}=m$. Inequality (3) for an indeterminate $q_{2}$ becomes $q_{2} \geq m n /(2 n-1)$ and is sharp for $n=1$. For values of $n>1$, we are not aware of values of $q_{2}$ with $\frac{m n}{2 n-1} \leq\left|q_{2}\right|<m$ that yield the desired surgery equivalence.

1.6. Organization. This article is organized into 4 sections. Section 2 discusses background material from Heegaard Floer homology. Section 3 is devoted to proving Theorem 1.3 and Proposition 1.6. The final Section 4 provides the missing calculations from the examples. 


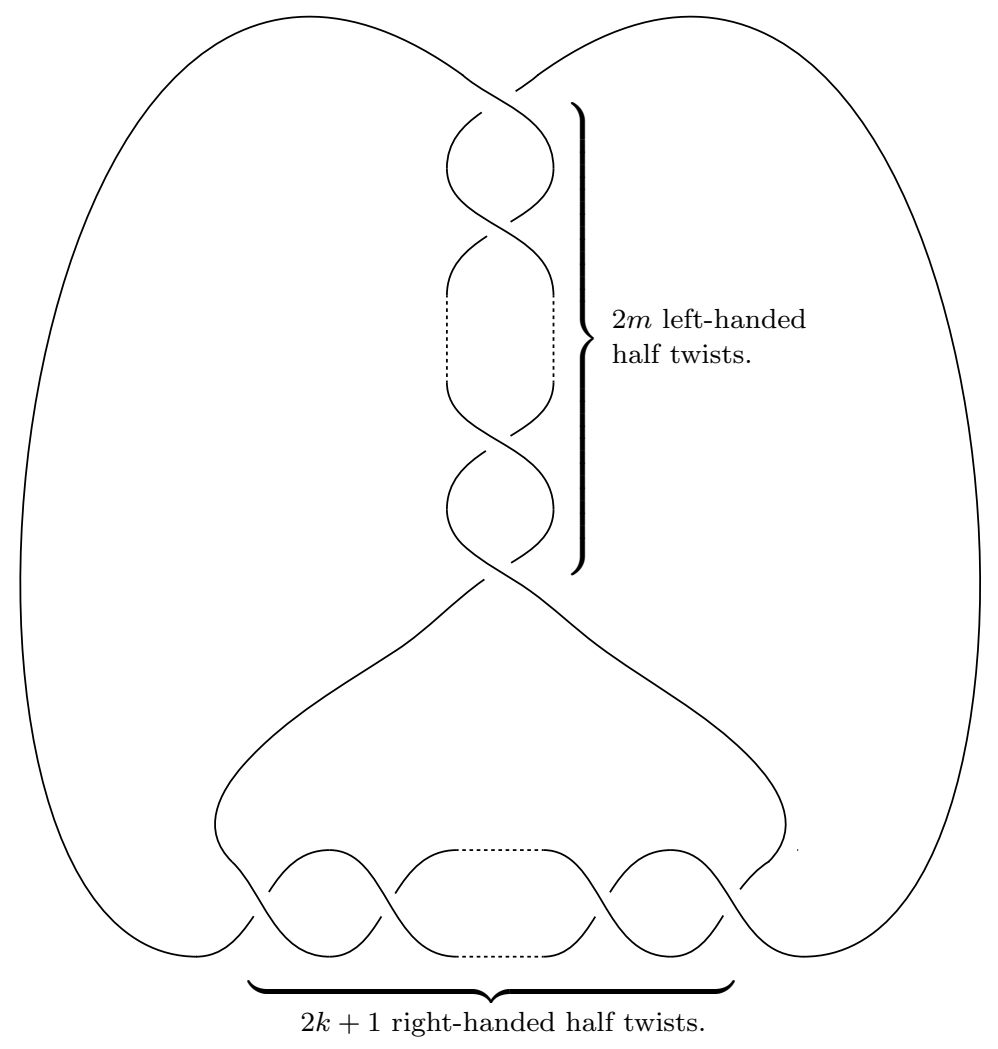

Figure 1 . The knot $K_{2 m, 2 k+1}$ with $m, k \in \mathbb{N}$.

\section{BACKGROUND MATERIAL}

2.1. Homology of mapping cones. This section gathers a few facts about the homology of the mapping cone of a chain map between two chain complexes. Let

$$
\begin{aligned}
\mathcal{C} & =\left\{\ldots \rightarrow C_{i+1} \stackrel{\partial_{i+1}}{\rightarrow} C_{i} \stackrel{\partial_{\xi}}{\rightarrow} C_{i-1} \rightarrow \ldots\right\}, \\
\mathcal{C}^{\prime} & =\left\{\ldots \rightarrow C_{i+1}^{\prime} \stackrel{\partial_{i+1}^{\prime}}{\rightarrow} C_{i}^{\prime} \stackrel{\partial_{i}^{\prime}}{\rightarrow} C_{i-1}^{\prime} \rightarrow \ldots\right\},
\end{aligned}
$$

be two finitely supported chain complexes of free Abelian groups. Let $f: \mathcal{C} \rightarrow \mathcal{C}^{\prime}$ be a chain map and let $f_{i}$ denote the restriction of $f$ to $C_{i}$.

Definition 2.1. The mapping cone of $f: \mathcal{C} \rightarrow \mathcal{C}^{\prime}$ is the complex

$$
\mathcal{M}=\left\{\ldots \rightarrow M_{i+1} \stackrel{D_{i+1}}{\longrightarrow} M_{i} \stackrel{D_{i}}{\longrightarrow} M_{i-1} \rightarrow \ldots\right\} \quad \text { with } \quad M_{i}=C_{i} \oplus C_{i+1}^{\prime},
$$

and with $D=\left\{D_{i}\right\}_{i \in \mathbb{N}}$ defined as

$$
D_{i}\left(c, c^{\prime}\right)=\left(\partial_{i} c, \partial_{i+1}^{\prime} c^{\prime}+(-1)^{i} f_{i}(c)\right), \quad\left(c, c^{\prime}\right) \in C_{i} \oplus C_{i+1}^{\prime} .
$$

It is easy to verify that the maps $\iota: \mathcal{C}^{\prime} \rightarrow \mathcal{M}$ and $\pi: \mathcal{M} \rightarrow \mathcal{C}$ defined by $\iota\left(c^{\prime}\right)=\left(0, c^{\prime}\right)$ and $\pi\left(c, c^{\prime}\right)=c$ are chain maps that fit into the short exact sequence

$$
0 \rightarrow C_{i+1}^{\prime} \stackrel{\iota}{\rightarrow} M_{i} \stackrel{\pi}{\rightarrow} C_{i} \rightarrow 0 .
$$


The connecting homomorphism $\delta_{i}: C_{i} \rightarrow C_{i}^{\prime}$ of this short exact sequence is given by $\delta_{i}=f_{i}$. This discussion implies the next, easy but useful, theorem:

Theorem 2.2. Let $\mathcal{M}$ be the mapping cone of $f: \mathcal{C} \rightarrow \mathcal{C}^{\prime}$. Then there is a long exact sequence

$$
\ldots \rightarrow H_{i+1}(\mathcal{C}) \stackrel{\left(f_{i+1}\right)_{*}}{\rightarrow} H_{i+1}\left(C^{\prime}\right) \stackrel{\iota_{*}}{\rightarrow} H_{i}(\mathcal{M}) \stackrel{\pi_{*}}{\rightarrow} H_{i}(\mathcal{C}) \stackrel{\left(f_{i}\right) *}{\rightarrow} H_{i}\left(\mathcal{C}^{\prime}\right) \rightarrow \ldots
$$

relating the homologies of $\mathcal{C}, \mathcal{C}^{\prime}$ and $\mathcal{M}$. In particular, if $\left(f_{i+1}\right)_{*}: H_{i+1}(\mathcal{C}) \rightarrow H_{i+1}\left(\mathcal{C}^{\prime}\right)$ is surjective then there is an isomorphism $H_{i}(\mathcal{M}) \cong \operatorname{Ker}\left(f_{i}\right)_{*}$.

2.2. The Heegaard Floer groups. In [12, 11] P. Ozsváth and Z. Szabó introduced chain complexes $C F^{\infty}(Y, \mathfrak{s}), C F^{ \pm}(Y, \mathfrak{s})$ and $\widehat{C F}(Y, \mathfrak{s})$ associated to the choice of a pointed Heegaard diagram $\left(\Sigma_{g},\left\{\alpha_{1}, \ldots, \alpha_{g}\right\},\left\{\beta_{1}, \ldots, \beta_{g}\right\}, z\right){ }^{1}$ for the closed and oriented 3-manifold $Y$, and a choice of $\operatorname{spin}^{c}$-structure $\mathfrak{s} \in \operatorname{Spin}^{c}(Y)$. Their homology groups $H F^{\infty}(Y, \mathfrak{s}), H F^{ \pm}(Y, \mathfrak{s})$ and $\widehat{H F}(Y, \mathfrak{s})$ are topological invariants of $(Y, \mathfrak{s})$ and are referred to as the Heegaard Floer homology groups of $(Y, \mathfrak{s})$.

The complex $C F^{\infty}(Y, \mathfrak{s})$ is freely generated by pairs $[x, i]$ with $i \in \mathbb{Z}$ and $x$ chosen from a finite set $\mathcal{X}$ determined by the Heegaard diagram, subject to the relation $\mathfrak{s}_{z}(x)=$ $\mathfrak{s}$, with $\mathfrak{s}_{z}: \mathcal{X} \rightarrow \operatorname{Spin}^{c}(Y)$ a function described in Section 2.6 of [12]. The complex comes equipped with an action of the polynomial ring $\mathbb{Z}[U]$ defined on generators by $U \cdot[x, i]=[x, i-1]$. The differential $\partial^{\infty}$ of this complex has the property that $\partial^{\infty}[x, i]$ is a sum of terms $[y, j]$ with $j \leq i$. Accordingly, the $\operatorname{subgroup~} C F^{-}(Y, \mathfrak{s})$ of $C F^{\infty}(Y, \mathfrak{s})$ generated by those $[x, i]$ with $i<0$ is a subcomplex. Their quotient complex is $C F^{+}(Y, \mathfrak{s})$, while $\widehat{C F}(Y, \mathfrak{s})$ is the kernel of the chain map $U: C F^{+}(Y, \mathfrak{s}) \rightarrow$ $C F^{+}(Y, \mathfrak{s})$. Alternatively, if we view $C F^{+}(Y, \mathfrak{s})$ as a $\mathbb{Z}$-filtered chain complex with filtration $\mathcal{F}_{+}([x, i])=i$, then $\widehat{C F}(Y, \mathfrak{s})=\mathcal{F}_{+}^{-1}(0)$.

When $c_{1}(\mathfrak{s})$ is a torsion element of $H^{2}(Y ; \mathbb{Z})$, the associated Heegaard Floer homology groups carry a $\mathbb{Q}$-grading and we write $H F_{(d)}^{\circ}(Y, \mathfrak{s})$ to distinguish the summand of $H F^{\circ}(Y, \mathfrak{s})$ in grading $d \in \mathbb{Q}$, with $\circ \in\{\infty, \pm, \widehat{ }\}$. We shall also write $H F^{\circ}(Y, \mathfrak{s})=$ $A_{\left(d_{1}\right)} \oplus B_{\left(d_{2}\right)} \oplus \ldots$ to express the same meaning, where $A, B, \ldots$ are Abelian groups, for instance $\widehat{H F}\left(S^{3}\right) \cong \mathbb{Z}_{(0)}$.

2.3. The knot Floer homology groups. Ozsváth and Szabó in [10] and J. Rasmussen [16] introduced chain complexes $C F K^{\infty}(Y, K, \mathfrak{t})$ and $\widehat{C F K}(Y, K, \mathfrak{t}, j)$ associated to a doubly pointed Heegaard diagram $\left(\Sigma_{g},\left\{\alpha_{1}, \ldots, \alpha_{g}\right\},\left\{\beta_{1}, \ldots, \beta_{g}\right\}, z, w\right)^{2}$ for the pair $(Y, K)$ consisting of a closed and oriented 3 -manifold $Y$ and a null homologous knot $K \subset Y$, along with the choice of a $\operatorname{spin}^{c}$-structure $\mathfrak{t} \in \operatorname{Spin}^{c}\left(Y_{0}(K)\right)$ (here $Y_{0}(K)$ denotes the manifold obtained by zero surgery on $K$ ) and an integer $j \in \mathbb{Z}$. Their homology groups $H F K^{\infty}(Y, K, \mathfrak{t})$ and $\widehat{H F K}(Y, K, \mathfrak{t}, j)$ are the knot Floer homology groups of $(Y, K, \mathfrak{t})$.

The complex $C F K^{\infty}(Y, K, \mathfrak{t})$ is freely generated by triples $[x, i, j]$ with $i, j \in \mathbb{Z}$ and with $x \in \mathcal{X}$ subject to the relation $\underline{\mathfrak{s}}(x)+(i-j) P D[\mu]=\mathfrak{t}$, where $\underline{\mathfrak{s}}: \mathcal{X} \rightarrow \operatorname{Spin}^{c}\left(Y_{0}(K)\right)$

\footnotetext{
${ }^{1}$ Here $z \in \Sigma_{g}$ is a point chosen in the complement of the $\alpha$ and $\beta$ attaching curves.

${ }^{2}$ Here $z, w \in \Sigma_{g}$ are points in the complement of the $\alpha$ and $\beta$ attaching curves, chosen with respect to the knot $K \subset Y$.
} 
is a function defined in Section 2.3 of [10], and $P D[\mu] \in H^{2}\left(Y_{0}(K) ; \mathbb{Z}\right)$ is the Poincaré dual of the meridian $\mu$ of $K$. This complex too has an action of $\mathbb{Z}[U]$ given on generators by $U \cdot[x, i, j]=[x, i-1, j-1]$, and its differential $\partial^{\infty}$ also has the property that $\partial^{\infty}[x, i, j]$ is a sum of terms $[y, k, \ell]$ with $k \leq i$ and $\ell \leq j$. Thus, the subgroups $C\{i \leq k\}$ and $C\{i \leq k, j \leq \ell\}$ generated by those $[x, i, j]$ with $i \leq k$, and $i \leq k$ and $j \leq \ell$ respectively, are subcomplexes of $C F K^{\infty}(Y, K, \mathfrak{t})$.

The function $\mathcal{F}_{K}: C F K^{\infty}(Y, K, \mathfrak{t}) \rightarrow \mathbb{Z}^{2}$ defined by $\mathcal{F}_{K}([x, i, j])=(i, j)$ renders $C F K^{\infty}(Y, K, \mathfrak{t})$ a $\mathbb{Z}^{2}$-filtered complex. This filtration induces a $\mathbb{Z}$-filtration $\mathcal{F}_{2}=\Pi_{2} \circ$ $\mathcal{F}_{K}$ (with $\Pi_{i}: \mathbb{Z}^{2} \rightarrow \mathbb{Z}$ being projection onto the $i$-th summand) on the quotient complex

$$
C\{i=0\}:=\frac{C\{i \leq 0\}}{C\{i \leq-1\}}
$$

The associated graded object of this filtered chain complex is $\widehat{C F K}(Y, K, \mathfrak{t}, m)$, that is

$$
\widehat{C F K}(Y, K, \mathfrak{t}, m)=\frac{\mathcal{F}_{2}^{-1}(\langle-\infty, m])}{\mathcal{F}_{2}^{-1}(\langle-\infty, m-1])} .
$$

The generators of $\widehat{C F K}(Y, K, \mathfrak{t}, m)$ are those $[x, 0, m]$ with $\underline{\mathfrak{s}}(x)=\mathfrak{t}+m P D[\mu]$.

Note that there is an isomorphism $\operatorname{Spin}^{c}\left(Y_{0}(K)\right) \cong \operatorname{Spin}^{c}(Y) \oplus \mathbb{Z}$ of affine spaces, which sends a $\operatorname{spin}^{c}$-structure $\mathfrak{t} \in \operatorname{Spin}^{c}\left(Y_{0}(K)\right)$ to a pair $(\mathfrak{s}, n)$ with $\mathfrak{s} \in \operatorname{Spin}^{c}(Y)$ obtained by the unique extension of $\left.\mathfrak{t}\right|_{Y_{0}(K)-K}$ to $Y$, and with $n=\frac{1}{2}\left\langle c_{1}(\mathfrak{t}),[\hat{F}]\right\rangle$ where $F \subset Y$ is a Seifert surface of $K$ and $\hat{F} \subset Y_{0}(K)$ is obtained from $F$ by capping it off with the meridional disk of the knot $\hat{K}$ which is the core of the solid torus filling. Under this isomorphism, the $\operatorname{Spin}^{c}(Y)$ component of $\mathfrak{s}(x)$ is $\mathfrak{s}_{z}(x)$.

The knot Floer homology chain complex $C F K^{\infty}(Y, K, \mathfrak{t})$ comes equipped with a "conjugation map", that is an isomorphism $J: C F K^{\infty}(Y, K, \mathfrak{t}) \rightarrow C F K^{\infty}(Y, K, \mathfrak{t})$ which commutes with $\partial^{\infty}$ and the action of $\mathbb{Z}[U]$. Formally, $J$ is induced by a reversal of the string orientation on $K$, but we shall not need this. The isomorphism $J$ induces an isomorphism (still denote by $J$ )

$$
J: C\{j=0\} \rightarrow C\{i=0\} .
$$

For this reason, one can compute $\widehat{H F K}(Y, K, \mathfrak{t}, m)$ from $C\{j=0\}$, viewed as a filtered complex (with filtration $[x, i, 0] \mapsto i$ ).

In our computations $J$ shall only play a secondary role, indeed, we shall only need to use the fact that $J$ is an isomorphism.

For later use, we define a sequence of special chain complexes extracted from $C F K^{\infty}(Y, K, \mathfrak{t})$. Let $k, \ell$ be two integers and let $C\{i \leq k, j \leq \ell\}$ be the subcomplex of $C F K^{\infty}(Y, K, \mathfrak{t})$ generated by those $[x, i, j]$ with $i \leq k$ and $j \leq \ell$. For $s \in \mathbb{Z}$, define the chain complexes $\hat{A}_{s}$ and $\hat{B}$ as

$$
\hat{A}_{s}=\frac{C\{i \leq 0, j \leq s\}}{C\{i \leq-1, j \leq s-1\}} \quad \text { and } \quad \hat{B}=C\{i=0\}
$$


These complexes come with accompanying chain maps $\hat{v}_{s}, \hat{h}_{s}: \hat{A}_{s} \rightarrow \hat{B}$ defined as

$$
\hat{v}_{s}([x, i, j])=\left\{\begin{array}{cl}
{[x, 0, j]} & ; i=0, \\
0 & ; i \neq 0,
\end{array} \quad \hat{h}_{s}([x, i, j])=\left\{\begin{array}{cl}
J([x, i-j, 0]) & ; j=s, \\
0 & ; j \neq s .
\end{array}\right.\right.
$$

Thus $\hat{v}_{s}$ is simply the projection map from $\hat{A}_{s}$ onto $\hat{B}$, cutting of the portion of $\hat{A}_{s}$ generated by those $[x, i, j]$ with $i<0$. Similarly, $\hat{h}_{s}$ is given by the action of $U^{-s}$, followed by projection onto $C\{j=0\}$, followed by $J$. We remark that $\hat{A}_{s} \cong \hat{B}$ whenever $s \geq g(K)$ in which case $\hat{v}_{s}$ is an isomorphism. In particular for all $s \geq g(K)$, $H_{*}\left(\hat{A}_{s}\right) \cong \widehat{H F}\left(S^{3}\right) \cong \mathbb{Z}$. Using the conjugation isomorphism $J$, one finds similarly that $H_{*}\left(\hat{A}_{s}\right) \cong \mathbb{Z}$ for all $s \leq-g(K)$ and that $\hat{h}_{s}$ is an isomorphism in this range. We shall rely on this facts tacitly going forward.

As was the case with Heegaard Floer groups, the knot Floer groups too carry a rational grading, provided $c_{1}(\mathfrak{s})$ is torsion (with $\mathfrak{t}=(\mathfrak{s}, m)$ ) and we shall similarly write, for example, $\widehat{H F K}_{(d)}(Y, K, \mathfrak{t}, m)$ to single out the grading $d$ term of $\widehat{H F K}(Y, K, \mathfrak{t}, m)$. Or we shall write $\widehat{H F K}(Y, K, \mathfrak{t}, m) \cong A_{\left(d_{1}\right)} \oplus B_{\left(d_{2}\right)} \oplus \ldots$ for the same thing.

Knot Floer homology of $(Y, K)$ can be thought of as a $\mathbb{Z}$-filtration on Heegaard Floer homology of $Y$. Namely, the projection $\Pi: C F K^{\infty}(Y, K, \mathfrak{t}) \rightarrow C F^{\infty}(Y, \mathfrak{s})$ (with $\mathfrak{t}=$ $(\mathfrak{s}, m)$ under the above isomorphism $\left.\operatorname{Spin}^{c}\left(Y_{0}(K)\right) \cong \operatorname{Spin}^{c}(Y) \oplus \mathbb{Z}\right)$ given on generators $\Pi([x, i, j])=[x, i]$, is an isomorphism of chain complexes, and the composition $\Pi_{1} \circ$ $\mathcal{F}_{K} \circ \Pi^{-1}: C F K^{\infty}(Y, \mathfrak{s}) \rightarrow \mathbb{Z}$ is a filtration on $C F^{\infty}(Y, \mathfrak{s})$. The same map renders $\widehat{C F}(Y, \mathfrak{s}), \hat{A}_{s}$ and $\hat{B}$ into $\mathbb{Z}$-filtered complexes. Applying the Leray spectral sequence to these filtered chain complexes, we find that

(i) There is a Leray spectral sequence whose $E^{2}$-term is isomorphic, as a $\mathbb{Z}[U]$ module, to $\widehat{H F K}(Y, K, \mathfrak{t}) \otimes_{\mathbb{Z}} \mathbb{Z}\left[U, U^{-1}\right]$, and that converges to $H F^{\infty}(Y, \mathfrak{s})$, and respects the rational gradings when $c_{1}(\mathfrak{s})$ is torsion. By $\widehat{H F K}(Y, K, \mathfrak{t})$ we mean $\oplus_{m \in \mathbb{Z}} \widehat{H F K}(Y, K, \mathfrak{t}, m)$.

(ii) There is a Leray spectral sequence whose $E^{2}$-term is isomorphic to $\widehat{H F K}(Y, K, \mathfrak{t})$ and that converges to $\widehat{H F}(Y, \mathfrak{s})$, and that respects the rational gradings when $c_{1}(\mathfrak{s})$ is torsion.

(ii') There is a Leray spectral sequence whose $E^{2}$-term is isomorphic to $\oplus_{j \in \mathbb{Z}} \widehat{H F K}(Y, K, \mathfrak{t}, j) \otimes$ $U^{j}$ and converges to $\widehat{H F}(Y, \mathfrak{s})$, and that respects the rational gradings when $c_{1}(\mathfrak{s})$ is torsion. This sequence is isomorphic to that from (ii) by using the isomorphism $J$ from (4).

(iii) There is a Leray spectral sequence whose $E^{2}$-term is isomorphic to

$$
\left(\bigoplus_{j \leq s} \widehat{H F K}(Y, K, \mathfrak{t}, j)\right) \oplus\left(\bigoplus_{j>s} \widehat{H F K}(Y, K, \mathfrak{t}, j) \otimes U^{j-s}\right)
$$

and that converges to $H_{*}\left(\hat{A}_{s}\right)$, and that respects the rational gradings when $c_{1}(\mathfrak{s})$ is torsion.

These spectral sequences are powerful computational tools that we shall heavily rely on. 
In the case of $Y=S^{3}$ we shall simplify notation and write $C F K^{\infty}(K)$ for $C F K^{\infty}\left(S^{3}, K, \mathfrak{t}_{0}\right)$ with $\mathfrak{t}_{0} \in \operatorname{Spin}^{c}\left(S_{0}^{3}((K))\right)$ characterized by $c_{1}\left(\mathfrak{t}_{0}\right)=0$. We shall also write $\widehat{C F K}(K, j)$ for $\widehat{C F K}\left(S^{3}, K, \mathfrak{t}_{0}, j\right)$, and we use similar notation for the homologies of these two chain complexes.

2.4. The rational surgery formula. This section describes the algorithm from [14] for the computation of $\widehat{H F}\left(S_{p / q}^{3}(K), \mathfrak{s}\right)$ for a knot $K$ in $S^{3}$.

For $i \in \mathbb{Z}$ let

$$
\hat{\mathbb{A}}_{i}=\oplus_{s \in \mathbb{Z}}\left(s, \hat{A}_{\left\lfloor\frac{i+p s}{q}\right\rfloor}\right) \quad \text { and } \quad \hat{\mathbb{B}}=\hat{\mathbb{B}}_{i}=\oplus_{s \in \mathbb{Z}}(s, \hat{B}) \cdot 3^{3}
$$

In the above, both $\left(k, \hat{A}_{\ell}\right)$ and $(k, \hat{B})$ denote copies of $\hat{A}_{\ell}$ and $\hat{B}$ respectively and $\lfloor x\rfloor$ is the largest integer smaller than or equal to $x$. We use the maps $\hat{v}_{k}, \hat{h}_{k}: \hat{A}_{k} \rightarrow \hat{B}$ to define maps $\hat{v}, \hat{h}: \hat{\mathbb{A}}_{i} \rightarrow \hat{\mathbb{B}}_{i}$ by the convenction that $\hat{v}$ maps $\left(s, \hat{A}_{\left\lfloor\frac{i+p s}{q}\right\rfloor}\right)$ to $(s, \hat{B})$ via $\hat{v}_{\left\lfloor\frac{i+p s}{q}\right\rfloor}$, while $\hat{h}$ maps $\left(s, \hat{A}_{\left\lfloor\frac{i+p s}{q}\right\rfloor}\right)$ to $(s-1, \hat{B})$ via $\hat{h}_{\left\lfloor\frac{i+p s}{q}\right\rfloor}$. Setting $r=p / q$, we define the chain map $\hat{D}_{i, r}: \hat{\mathbb{A}}_{i} \rightarrow \hat{\mathbb{B}}_{i}$ as

$$
\hat{D}_{i, r}\left(\left\{\left(s, a_{s}\right)\right\}_{s \in \mathbb{Z}}\right)=\left\{\left(s, b_{s}\right)\right\}_{s \in \mathbb{Z}} \quad \text { with } \quad b_{s}=\hat{v}_{\left\lfloor\frac{i+p s}{q}\right\rfloor}\left(a_{s}\right)+\hat{h}_{\left\lfloor\frac{i+p(s-1)}{q}\right\rfloor}\left(a_{s-1}\right) .
$$

Let $\hat{\mathbb{X}}_{i, r}$ be the mapping cone of $\hat{D}_{i, r}$. Note that that $\mathbb{X}_{i, r}=\mathbb{X}_{i^{\prime}, r}$ whenever $i$ and $i^{\prime}$ are congruent modulo $p$. Given this we further modify our notation to $\hat{\mathbb{X}}_{[i], r}$ where $[i] \in \mathbb{Z} / p \mathbb{Z}$ is the equivalence class of $i \in \mathbb{Z}$ modulo $p$.

Theorem 2.3 (Ozsváth-Szabó [14]). Let $K \subset S^{3}$ be a knot and let $p, q \in \mathbb{Z}$ be a pair of relatively prime, nonzero integers. Then there is an affine identification of $\operatorname{Spin}^{c}\left(S_{p / q}^{3}(K)\right)$ with $\mathbb{Z} / p \mathbb{Z}$, with respect to which there is an isomorphism

$$
\widehat{H F}\left(S_{p / q}^{3}(K), \mathfrak{s}\right) \cong H_{*}\left(\hat{\mathbb{X}}_{[i], r}\right)
$$

Theorem 2.3 is the main instrument for the proofs of our results, and we pause before proceeding to give a simple example. The key ingredient to using Theorem 2.3 is an understanding of the groups $H_{*}\left(\hat{A}_{s}\right)$ (the homology $H_{*}(\hat{B})$ is isomorphic to $\mathbb{Z}_{(0)}$ for knots in $S^{3}$ ) and the maps $\hat{v}_{s}, \hat{h}_{s}$. We shall get a handle on both by using of the Leray spectral sequences from Section 2.3 .

To begin with, we introduce a useful way of conceptualizing the chain complex $C F K^{\infty}(K)$. Namely, we represent each of the groups $\widehat{H F K}(K, j)$ by a dot in a coordinate plane, placed at the point $(0, j)$. We let $U$ act by translation by the vector $(-1,-1)$, and thus fill out a diagonal region of the plane with dots representing the groups $\widehat{H F K}(K, j) \otimes U^{m}$ (the latter sitting at coordinates $(-m,-m+j)$ ). We shall refer to the horizontal coordinate as the $i$-coordinate and the vertical one as the $j$ coordinate. Thus, the groups in the entire $i j$-plane are the $E^{2}$ term of the Leray spectral sequence converging to $H F^{\infty}\left(S^{3}\right)$, the $j$-axis contains the $E^{2}$ term converging to $\widehat{H F}\left(S^{3}\right)$ and the "angle" of points $(i, j)$ with $\min (i, j-s)=0$ represents the $E^{2}$ term

\footnotetext{
${ }^{3}$ The index $i$ in $\hat{\mathbb{B}}_{i}$ if for bookkeeping purposes only, the complex $\hat{\mathbb{B}}_{i}$ is independent of $i$. When convenient we will write $\hat{\mathbb{B}}$ instead of $\hat{\mathbb{B}}_{i}$.
} 
converging to $H_{*}\left(\hat{A}_{s}\right)$. Knowing the $E^{\infty}$ terms of the first two sequences typically lets one pin down the differentials of the higher order terms of the spectral sequence, and use the third of these sequences to compute $H_{*}\left(\hat{A}_{s}\right)$. Here is an example.

Example 2.4. Consider the $(3,4)$ torus knot $K=T_{(3,4)}$. It's knot Floer homology can be computed from the results of [13]:

$$
\widehat{H F K}\left(T_{(3,4)}, j\right) \cong\left\{\begin{array}{cl}
\mathbb{Z}_{(0)} & ; j=3, \\
\mathbb{Z}_{(-1)} & ; j=2, \\
\mathbb{Z}_{(-2)} & ; j=0, \\
\mathbb{Z}_{(-5)} & ; j=-2, \\
\mathbb{Z}_{(-6)} & ; j=-3 .
\end{array}\right.
$$

The spectral sequence (ii) from Section 2.3 converging to $\widehat{H F}\left(S^{3}\right) \cong \mathbb{Z}_{(0)}$ shows that there are two "vertical" higher differentials on its $E^{2}$ term, namely $d_{2}: \mathbb{Z}_{(-1)} \rightarrow \mathbb{Z}_{(-2)}$ and $d_{2}: \mathbb{Z}_{(-5)} \rightarrow \mathbb{Z}_{(-6)}$, both isomorphisms, and the spectral sequence abuds after this level. From the spectral sequence (ii'), we find similar "horizontal" differentials. Finally, applying sequence (i), shows that there are no further "diagonal" differentials in the $i j$-plane, see Figure 2 .

Having used spectral sequences (i), (ii) and (ii') from Section 2.3 to pin down the differentials in the sequence (i), we now turn to the spectral sequence (iii) to compute the homologies $H_{*}\left(\hat{A}_{s}\right)$. Using Figure 2 , it is now easy to find that $H_{*}\left(\hat{A}_{s}\right) \cong \mathbb{Z}$ for all $s \in \mathbb{Z}$ and that

$$
\hat{v}_{s}=\left\{\begin{array}{cl}
\text { id } & ; \quad s \geq 3, \\
0 & ; \quad s \leq 2,
\end{array} \quad \text { and } \quad \hat{h}_{s}=\left\{\begin{array}{cl}
0 & ; \quad s \geq-2, \\
\text { id } \quad ; & s \leq-3 .
\end{array}\right.\right.
$$

With these in place, it is now easy to use Theorem 2.3. We invite the reader to check that for instance $\widehat{H F}\left(S_{-1}^{3}\left(T_{(3,4)}\right)\right) \cong \mathbb{Z}^{11}$.

2.5. The Ozsváth-Szabó $\tau$-invariant. In [9] Ozsváth and Szabó introduced a concordance invariant $\tau(K)$ for a knot $K$ in $S^{3}$. It is defined as

$$
\left.\tau(K)=\min \left\{j \in \mathbb{Z} \mid\left(\iota_{j}\right)_{*}: H_{*}\left(\mathcal{F}_{K}^{-1}(-\infty, j]\right)\right) \rightarrow \widehat{H F}\left(S^{3}\right) \text { is nontrivial. }\right\},
$$

where $\left.\iota_{j}: \mathcal{F}_{K}^{-1}(-\infty, j]\right) \rightarrow \widehat{C F}\left(S^{3}\right)$ is the inclusion map. The definition is well posed as $\iota_{j}$ is an isomorphism for all sufficiently large $j$.

\section{Proofs}

3.1. Proof of Theorem 1.3. As in the setup of Theorem 1.3, let $K \subset S^{3}$ be a knot of genus $g \geq 1$, and let $p, q$ be two nonzero and relatively prime integers with $p>0$. Let $Y=S_{p / q}^{3}(K)$ be the results of $p / q$-framed Dehn surgery on $K$ and let $\ell$ be the number of $L$-structures on $Y$. Recalling the identification $\operatorname{Spin}^{c}\left(S_{p / q}^{3}(K)\right)$ with $\mathbb{Z} / p \mathbb{Z}$ from Theorem 2.3, we shall label $\operatorname{spin}^{c}$-structures on $S_{p / q}^{3}(K)$ by $[i]$, the equivalence class in $\mathbb{Z} / p \mathbb{Z}$ of the integer $i$. Our goal then is to demonstrate the validity of the inequality

$$
2 g-1 \geq \frac{p-\ell}{|q|}
$$




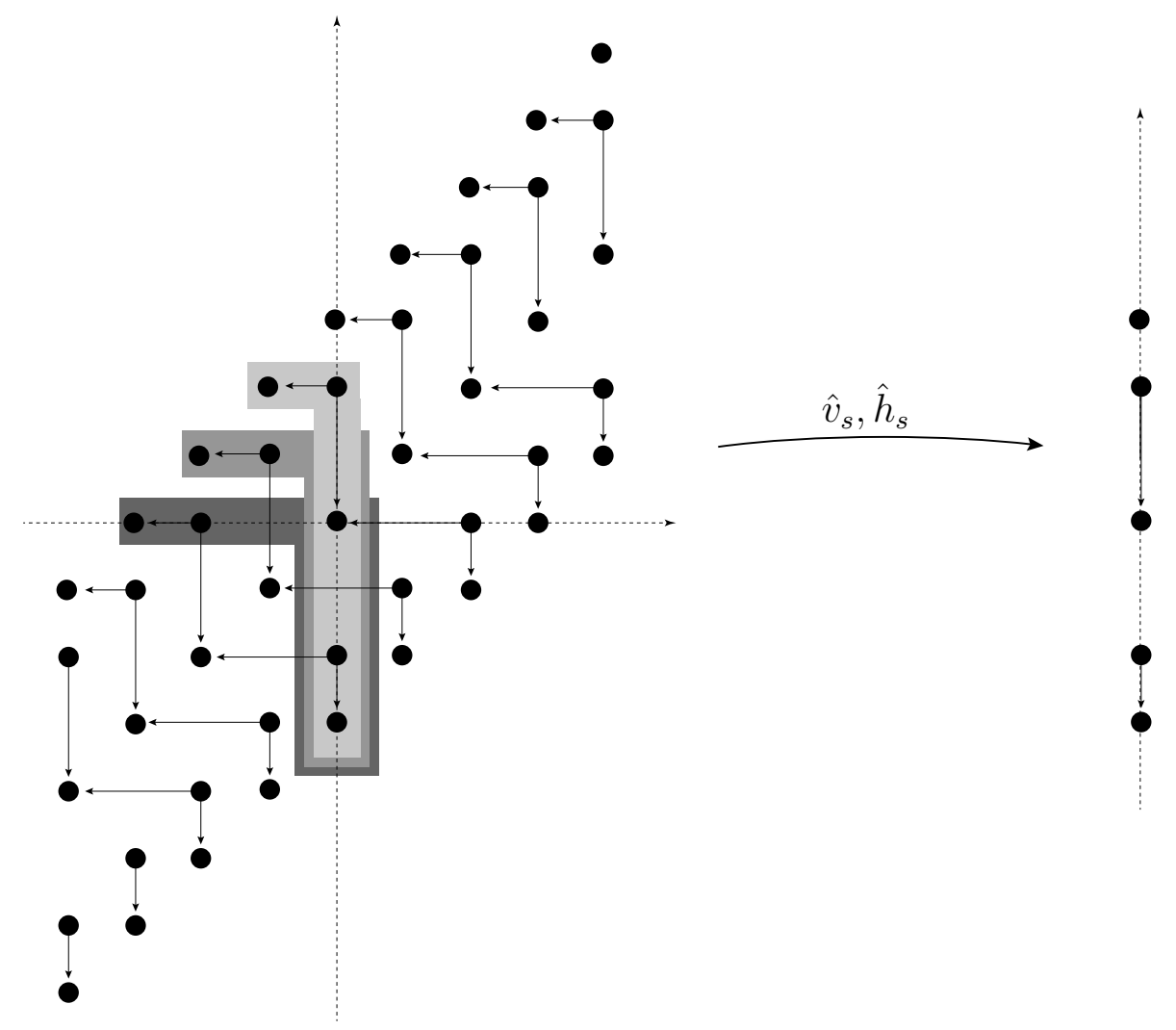

Figure 2. Example of the $(3,4)$-torus knot. The homology of the shaded regions give $H_{*}\left(\hat{A}_{s}\right) \cong \mathbb{Z}$ for $s=2$ (lightest shading), $s=1$ (medium shading) and $s=0$ (darkest shading). In each case $\hat{v}_{s}=0$ and $\hat{h}_{s}=0$.

We note firstly that is suffices to establish (7) for $q>0$. For if $q<0$ and $Y=S_{p / q}^{3}(K)$, then $-Y=S_{-p / q}^{3}(\bar{K})$ where $\bar{K}$ is the mirror image of $K$. The genus of $\bar{K}$ equals that of $K$, and the number of $L$-structures on $-Y$ equals that on $Y$ [11]. Thus, inequality (7) for $p / q$-surgery on $K$ is established by establishing it for $-p / q$-surgery on $\bar{K}$.

Assuming $p, q>0$, we prove Theorem 1.3 following a twofold strategy:

(a) We shall first count the number of $\operatorname{spin}^{c}$-structures $[i] \in \operatorname{Spin}^{c}\left(S_{p / q}^{3}(K)\right)$ for which either $\left\lfloor\frac{i+p s}{q}\right\rfloor \geq g$ or $\left\lfloor\frac{i+p s}{q}\right\rfloor \leq-g$ for all values $s \in \mathbb{Z}$, and show that there is $\max (p-(2 g-1) q, 0)$ such $\operatorname{spin}^{c}$-structures.

(b) We shall show that each $\operatorname{spin}^{c}$-structure $[i] \in \operatorname{Spin}^{c}\left(S_{p / q}^{3}(K)\right)$ from part (a) is an $L$-structure.

Put together, these two claims show that the number $\ell$ of $L$-structures on $Y$ satisfies the inequality

$$
\ell \geq\left\{\begin{array}{cc}
p-(2 g-1) q & ; \quad p-(2 g-1) q \geq 0, \\
0 & ; \quad p-(2 g-1) q \leq 0 .
\end{array}\right.
$$

If $p-(2 g-1) q \geq 0$ then the inequality $\ell \geq p-(2 g-1) q$ readily transforms into inequality (7). While the inequality $\ell \geq 0$ is without content, it occurs when $p-(2 g-1) q \leq 0$ 
giving $2 g-1 \geq p / q$ and clearly $p / q \geq(p-\ell) / q$, establishing (7) once more. With this understood, we turn to proving Claims (a) and (b).

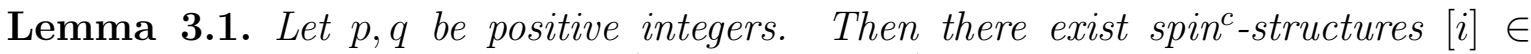
$\operatorname{Spin}^{c}\left(S_{p / q}^{3}(K)\right)$ for which either $\left\lfloor\frac{i+p s}{q}\right\rfloor \geq g$ or $\left\lfloor\frac{i+p s}{q}\right\rfloor \leq-g$ for all values $s \in \mathbb{Z}$, precisely when $p>(2 g-1) q$. If this inequality is met, then the said spin ${ }^{c}$-structures $[i]$ are the equivalence classes of the set $\{g q, \ldots, p+q-g q-1\}$. In particular there are $p-(2 g-1) q$ such spin ${ }^{c}$-structures.

Proof. Let $[i] \in \mathbb{Z} / p \mathbb{Z}$ be a $\operatorname{spin}^{c}$-structure, and for simplicity let us agree to choose $i$ from the set $\{0, \ldots, p-1\}$. Since $p$ and $q$ have been assumed to be positive, then the function $s \mapsto\left\lfloor\frac{i+p s}{q}\right\rfloor$ is non-decreasing. Additionally, note that $\left\lfloor\frac{i+p s}{q}\right\rfloor<0$ for $s<0$ and $\left\lfloor\frac{i+p s}{q}\right\rfloor \geq 0$ for $s \geq 0$. Accordingly,

$$
\left\lfloor\frac{i+p s}{q}\right\rfloor \leq\left\lfloor\frac{i-p}{q}\right\rfloor \text {, for all } s \leq-1 \quad \text { and } \quad\left\lfloor\frac{i+p s}{q}\right\rfloor \geq\left\lfloor\frac{i}{q}\right\rfloor \text {, for all } s \geq 0 .
$$

Therefore, if $i \in\{0, \ldots, p-1\}$ is such that $\left\lfloor\frac{i-p}{q}\right\rfloor \leq-g$ and $\left\lfloor\frac{i}{q}\right\rfloor \geq g$, it will satisfy the requirement of the lemma. The set of $i \in\{0, \ldots, p-1\}$ that obey the inequality $\left\lfloor\frac{i}{q}\right\rfloor \geq g$ is $\{g q, \ldots, p-1\}$ if $g q \leq p-1$ (and is otherwise empty), while the set of those $i$ for which $\left\lfloor\frac{i-p}{q}\right\rfloor \leq-g$, is given by $\{0, \ldots, p+q-g q-1\}$ if $p+q-g p-1 \geq 0$ (and is otherwise empty). The intersection of these two sets is $\{g q, \ldots, p+q-g q-1\}$ which has cardinaliy $p-(2 g-1) q$, and is nonempty if and only if $p-1 \geq g q$ and $p+q-1 \geq g q$ and $p+q>2 g q$. The third of these inequalities implies the first two, and the claim of the lemma follows.

Lemma 3.2. Let $K \subset S^{3}$ be a knot of genus $g \geq 1$ and let $p, q$ be relatively prime integers. Let $[i] \in \operatorname{Spin}^{c}\left(S_{p / q}^{3}(K)\right)$ be a spinc-structure on $Y=S_{p / q}^{3}(K)$ for which either $\left\lfloor\frac{i+p s}{q}\right\rfloor \geq g$ or $\left\lfloor\frac{i+p s}{q}\right\rfloor \leq-g$ for all values $s \in \mathbb{Z}$. Then $[i]$ is an L-structure.

Proof. Assume firstly that $p, q$ are positive. For convenience let us choose $i$ from the set $\{0, \ldots, p-1\}$ so that the assumption of the lemma leads to $\left\lfloor\frac{i+p s}{q}\right\rfloor \geq g$ for all $s \geq 0$ and $\left\lfloor\frac{i+p s}{q}\right\rfloor \leq-g$ for all $s<0$. Accordingly,

$$
H_{*}\left(\hat{A}_{\left\lfloor\frac{i+p s}{q}\right\rfloor}\right) \cong\left\{\begin{array}{cc}
H_{*}(C\{i=0\}) \cong \mathbb{Z} & ; s \geq 0, \\
H_{*}\left(C\left\{j=\left\lfloor\frac{i+p s}{q}\right\rfloor\right\}\right) \cong \mathbb{Z} \quad ; \quad s<0 .
\end{array}\right.
$$

Additionally, the maps in homology induced by $\hat{v}_{k}$ and $\hat{h}_{k}$ (still denoted $\hat{v}_{k}$ and $\hat{h}_{k}$ ) are given by

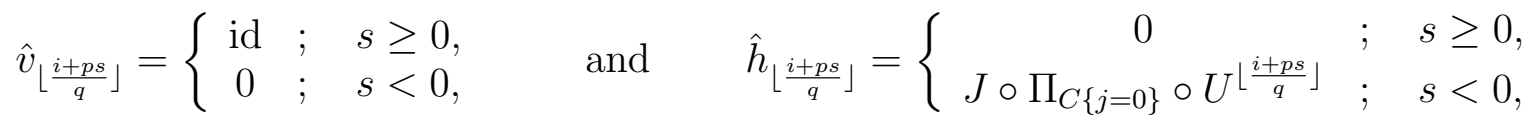

with $\Pi_{C\{j=0\}}$ being projection onto $C\{j=0\}$. Though we may not know the map $J$ explicitly, we note that $\hat{h}_{\left\lfloor\frac{i+p s}{q}\right\rfloor}$ is an isomorphism for all $s<0$.

Consider now the mapping cone $\hat{\mathbb{X}}_{[i], r}($ with $r=p / q)$ of $\hat{v}+\hat{h}: \hat{\mathbb{A}}_{i} \rightarrow \hat{\mathbb{B}}_{i}$. The explicit formulae for $\hat{v}$ and $\hat{h}$ above show that the map in homology induced by $\hat{v}+\hat{h}$ is onto, 
and so in light of Theorem 2.2 we obtain the isomorphism

$$
\widehat{H F}\left(S_{p / q}^{3}(K),[i]\right) \cong H_{*}\left(\hat{\mathbb{X}}_{[i], r}\right) \cong \operatorname{Ker}\left(\hat{v}+\hat{h}: H_{*}\left(\hat{\mathbb{A}}_{i}\right) \rightarrow H_{*}\left(\hat{\mathbb{B}}_{i}\right)\right)
$$

The kernel of $\hat{v}+\hat{h}$ is easily computed. Namely, consider the following diagram in which the vertical maps indicate the nonzero $\hat{v}_{k}$ 's and the slanted maps correspond to the nonzero $\hat{h}_{k}$ 's:

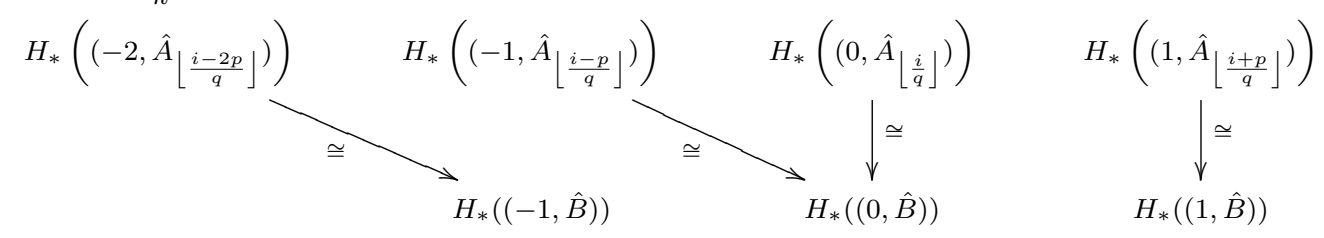

The direct sum of the groups in the top row (which is infinite in both directions) represents $H_{*}\left(\hat{\mathbb{A}}_{i}\right)$ while the direct sum of the groups in the bottom row (likewise infinite in both directions) represents $H_{*}\left(\hat{\mathbb{B}}_{i}\right)$. The kernel of $\hat{v}+\hat{h}$ is easily explicitly identified as

$$
\operatorname{Ker}(\hat{v}+\hat{h})=\left\{\left\{\left(s, a_{\left\lfloor\frac{i+p s}{q}\right\rfloor}\right)\right\}_{s \in \mathbb{Z}} \mid a_{\left\lfloor\frac{i+p s}{q}\right\rfloor}=0 \text { for } s \neq-1,0 \text { and } a_{\left\lfloor\frac{i-p}{q}\right\rfloor}+a_{\left\lfloor\frac{i}{q}\right\rfloor}=0\right\} .
$$

Clearly $\operatorname{Ker}(\hat{v}+\hat{h}) \cong \mathbb{Z}$ as needed.

In the case where $p / q<0$, the above argument needs slight modification. Specifically, the homology of $\hat{\mathbb{X}}_{[i], r}$ is computed as the homology of the mapping cone

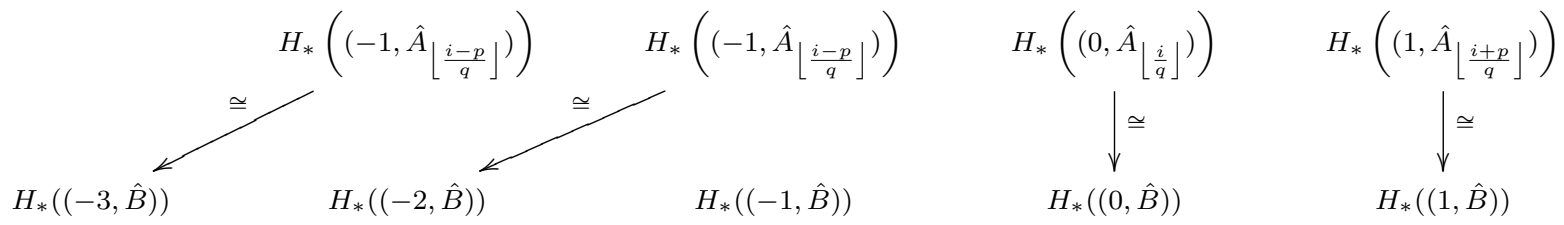

This time $\hat{v}+\hat{h}$ is into, rather than being onto, and an application of Theorem 2.2 shows that $H_{*}\left(\hat{\mathbb{X}}_{[i], r}\right) \cong \operatorname{Coker}(\hat{v}+\hat{h}) \cong H_{*}((-1, \hat{B})) \cong \mathbb{Z}$.

3.2. Proof of Proposition 1.6. Let $K$ be a knot of genus $g>1$ and assume firstly that $\tau(K)=g$. Let $p, q>0$ be relatively prime integers with $p>(2 g-1) q$. This inequality assures that all $\operatorname{spin}^{c}$-structures $[i]$ satisfy one of two properties:

- Either $\left\lfloor\frac{i+p s}{q}\right\rfloor \leq-g$ or $\left\lfloor\frac{i+p s}{q}\right\rfloor \geq g$ for all $s \in \mathbb{Z}$.

- There exists exactly one $s_{i} \in \mathbb{Z}$ such that $-g<\left\lfloor\frac{i+p s_{i}}{q}\right\rfloor<g$.

Spin $^{c}$-structures of the first kind are $L$-structures and there are exactly $p-(2 g-1) q$ of them (Lemmas 3.1 and 3.2). Turning to $\operatorname{spin}^{c}$-structures $[i]$ of the second kind, we note that the assumption of $\tau(K)=g$ implies the vanishing of certain of the maps $\hat{v}_{s}$ and $\hat{h}_{s}$. Namely, consider the factorization $\hat{v}_{s}=\iota_{s} \circ \pi_{s}$

$$
\hat{A}_{s} \stackrel{\pi_{s}}{\longrightarrow} \mathcal{F}_{K}^{-1}(\langle-\infty, s]) \stackrel{\iota_{s}}{\longrightarrow} \hat{B}=\widehat{C F}\left(S^{3}\right)
$$

with $\pi_{s}$ being the projection and $\iota_{s}$ the inclusion map. Since $\tau(K)=g$, it follow that $\hat{v}_{s}=0$ for all $s<g$ (since $\iota_{s}=0$ for $s<g$ ) and similarly that $\hat{h}_{s}=0$ for all $s>-g$. Of course, $\hat{v}_{s}$ is an isomorphism for all $s \geq g$ and $\hat{h}_{s}$ is an isomorphism for all $s \leq-g$. 
Accordingly, the homology of $\hat{\mathbb{X}}_{[i], r}$ (with $r=-p / q$ ) is the homology of the mapping cone

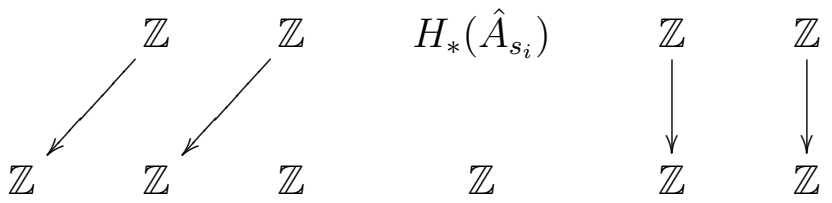

Theorem 2.2 implies then that $\mathbb{Z}^{2}$ injects into $H_{*}\left(\hat{\mathbb{X}}_{[i], r}\right)$ and so $[i]$ is not an $L$-structure.

The case of $\tau(K)=-g$ and $r=p / q$ follows by symmetry since $\widehat{H F}\left(S_{p / q}^{3}(K)\right) \cong$ $\widehat{H F}\left(S_{-p / q}^{3}(\bar{K})\right)$ and $\tau(\bar{K})=-\tau(K)$.

\section{EXAMPLES}

In this section we provide computations supporting our claims in Examples 1.8 1.10 from the introduction. The main tools are Theorems 2.2 and 2.3 which are used to compute the Heegaard Floer homology of a rational surgery $S_{p / q}^{3}(K)$ on a knot $K$, with the homologies $H_{*}\left(\hat{A}_{s}\right)$ and the maps $\hat{v}_{s}, \hat{h}_{s}: H_{*}\left(\hat{A}_{s}\right) \rightarrow H_{*}(\hat{B})$ as input. The latter groups and maps are computed with the help of the spectral sequences (i)-(iii) from Section 2.3.

Example 1.7 follows directly from Proposition 1.6 and the fact that $\tau\left(T_{(2,2 g+1)}\right)=g$.

4.1. Computations for Example 1.8. For a positive integer $n$, let $Y_{n}$ be the result of $-\frac{4 n+1}{n}$-framed surgery on the Figure Eight knot. The $\operatorname{spin}^{c}$-structures on $Y_{n}$ can be grouped into two disjoint categories:

- Spin $^{c}$-structures $[i] \in \mathbb{Z} /(4 n+1) \mathbb{Z}$ for which either $\left\lfloor\frac{i+(4 n+1) s}{n}\right\rfloor \leq-1$ or $\left\lfloor\frac{i+(4 n+1) s}{n}\right\rfloor \geq$ 1 for all $s \in \mathbb{Z}$.

- Spin ${ }^{c}$-structures $[i] \in \mathbb{Z} /(4 n+1) \mathbb{Z}$ for which there exists a unique $s_{i} \in \mathbb{Z}$ such that $\left\lfloor\frac{i+(4 n+1) s_{i}}{n}\right\rfloor=0$.

According to Lemma 3.1 there are $3 n+1$ spin $^{c}$-structures of the first kind, and each of them is an $L$-structure. To show that $\ell\left(Y_{n}\right)=3 n+1$, we need to demonstrate that none of the $\operatorname{spin}^{c}$-structures of the second kind is in an $L$-structure. This becomes an explicit calculation, after determining the differentials in the spectral sequence (i) from Section 2.3. Since the Figure Eight knot $4_{1}$ is alternating, with Alexander polynomial $t-3+t^{-1}$, and with vanishing signature, its knot Floer homology is given by

$$
\widehat{\operatorname{HFK}}\left(4_{1}, j\right) \cong \begin{cases}\mathbb{Z}_{(1)} & ; \quad j=1, \\ \mathbb{Z}_{(0)}^{3} & ; \quad j=0, \\ \mathbb{Z}_{(-1)} & ; \quad j=-1\end{cases}
$$

There is a pair of non vanishing vertical differentials $d_{2}: \mathbb{Z}_{(1)} \rightarrow \mathbb{Z}_{(0)}^{3}$ and $d_{2}: \mathbb{Z}_{(0)}^{3} \rightarrow$ $\mathbb{Z}_{(-1)}$ given by the inclusion into the first coordinate, and projection onto the third coordinate, respectively. Their horizontal counterparts looks as in Figure 6 and there are no other differentials. 


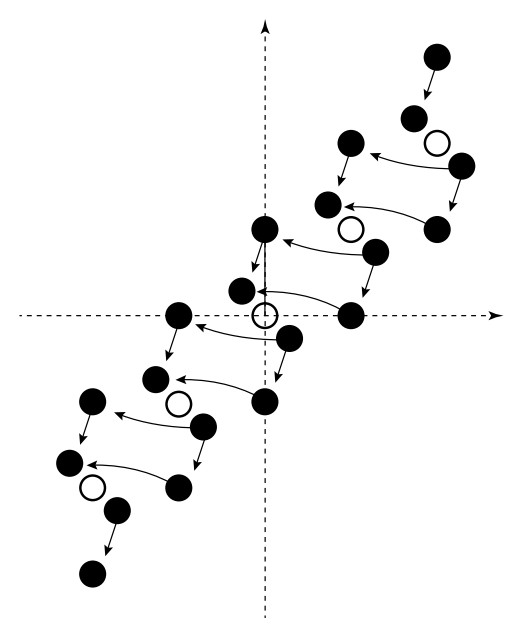

Figure 3. The differentials in the spectral sequence $E^{2}=\widehat{H F}\left(4_{1}\right) \otimes_{\mathbb{Z}}$ $\mathbb{Z}\left[U, U^{-1}\right]$ converging to $E^{\infty}=H F^{\infty}\left(S^{3}\right)$. The white dot represents the summand $\mathbb{Z}_{(0)}$ (and its various translates by $U^{n}$ ) containing the generator of $\widehat{H F}\left(S^{3}\right)$, the two blacks dots right next to it represent the other two copies of $\mathbb{Z}_{(0)}$ in $\widehat{H F K}\left(4_{1}, 0\right)$ (and their various translates by $U^{n}$ ).

From this, it is now an easy matter to find that

$$
H_{*}\left(\hat{A}_{s}\right) \cong\left\{\begin{array}{cl}
\mathbb{Z} & ; s \neq 0, \\
\mathbb{Z} \oplus \mathbb{Z}^{2} & ; s=0,
\end{array} \quad \hat{v}_{s}=\left\{\begin{array}{cl}
\text { id } & ; s>0, \\
\pi_{1} & ; s=0, \\
0 & ; s<0,
\end{array} \quad \hat{h}_{s}=\left\{\begin{array}{cl}
0 & ; s>0, \\
\pi_{1} & ; s=0, \\
\text { id } & ; s<0 .
\end{array}\right.\right.\right.
$$

In the above, $\pi_{1}: \mathbb{Z} \oplus \mathbb{Z}^{2} \rightarrow \mathbb{Z}$ is the projection on the first summand. These computations show that $\widehat{H F}(Y,[i]) \cong \mathbb{Z}^{3}$ for all spin $^{c}$-structures $[i]$ of the second kind, as this Heegaard Floer group is the homology of the mapping cone below (with arrows without labels corresponding to isomorphisms).

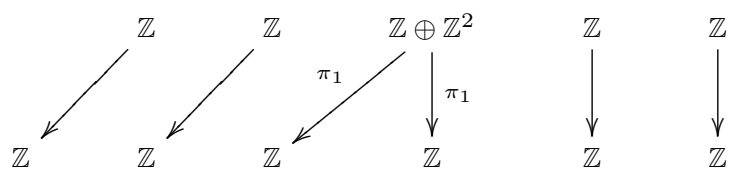

It follows that $\ell\left(Y_{n}\right)=3 n+1$ as claimed, demonstrating the sharpness of inequalities (11) and (2) from Theorem 1.3 and Corollary 1.4 (in the case of $q=1$ ) respectively. In particular, $g_{\mathbb{Q}}\left(Y_{n}\right)=1$ and $g_{\mathbb{Z}}\left(Y_{n}\right) \geq \frac{n+1}{2}$ and hence $g_{\mathbb{Z}}\left(Y_{n}\right)-g_{\mathbb{Q}}\left(Y_{n}\right) \geq \frac{n-1}{2}$.

To show that $g_{\mathbb{Z}}\left(Y_{n}\right)$ is finite, we show that $Y_{n}$ also arises as an integral surgery on a knot. We will do so by applying a set of Rolfsen twists [17, 18] to the framed knot $\left(4_{1},-\frac{4 n+1}{n}\right)$ through which $Y_{n}$ was defined.

Consider first the framed link from Figure 4(a). After applying a Rolfsen twist to its component with framing 1 (and after discarding the resulting $\infty$-framed unknot) we arrive at the framed knot in Figure $4(\mathrm{~b})$, yielding $\left(4_{1},-\frac{4 n+1}{n}\right)$ after a simple isotopy. Thus $Y_{n}$ is also the result of Dehn surgery on the framed link in Figure 4(a). 


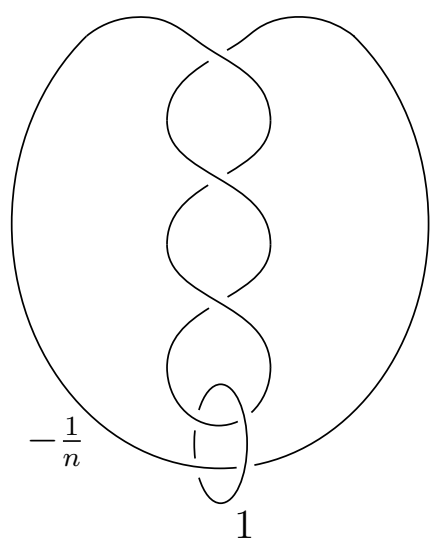

(a)

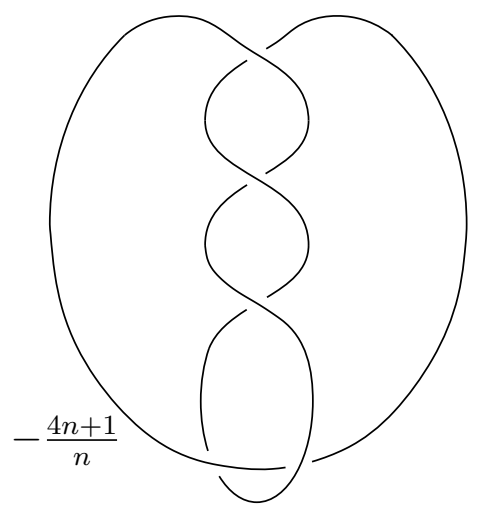

(b)

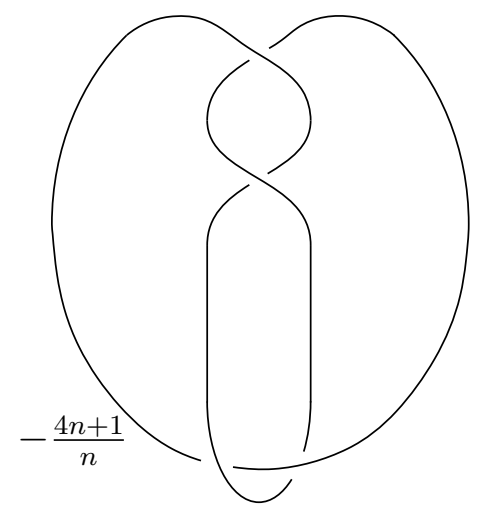

(c)

Figure 4. A Rolfsen twist on the 1-framed unknot in Figure (a) yields the framed knot in Figure (b). Figure (c) results from the latter by a simple isotopy.

Applying an isotopy to the framed link from Figure 4(a) gives the framed link in Figure 5(a). The latter, after performing a Rolfsen twist on the $-\frac{1}{n}$-framed component (and again discarding the resulting $\infty$-framed unknot) leads to the framed knot in Figure 5(b). The framing of the latter is an integer, showing that $g_{\mathbb{Z}}\left(Y_{n}\right)$ is finite.

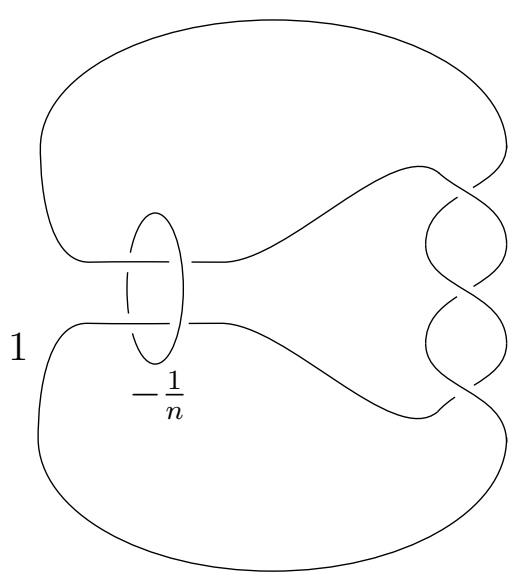

(a)

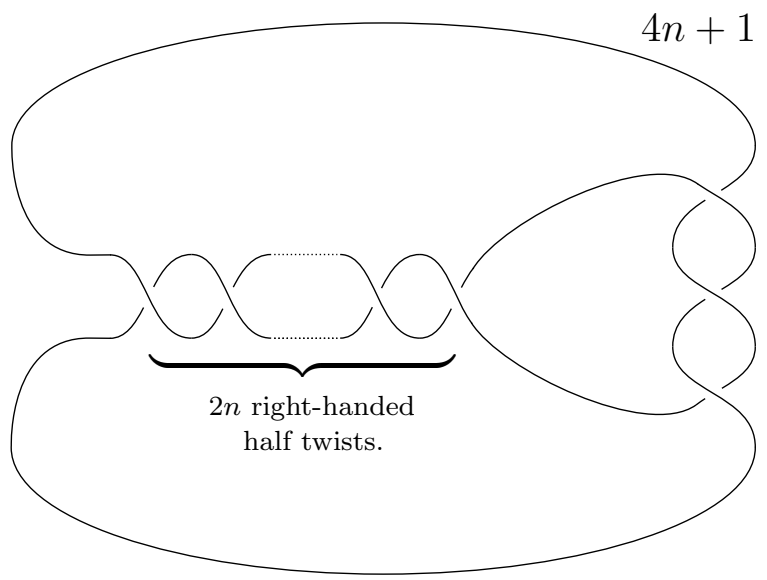

(b)

Figure 5. Applying a Rolfsen twist to the $-\frac{1}{n}$-framed component of the link in Figure (a), yields the framed knot in Figure (b).

4.2. Computations for Example 1.10. Let $K_{2 m, 2 k+1}$ be the knot as defined by Figure 1 , with $m, k \in \mathbb{N}$. It is easy to establish that $K_{2 m, 2 k+1}$ is an alternating knot, 
with signature $2 m$, and with Alexander polynomial

$$
\Delta_{2 m, 2 k+1}(t)=(k+1)\left(t^{m}+t^{-m}\right)-(2 k+1) \sum_{i=0}^{2 m-2}(-1)^{i} t^{m-1-i},
$$

from which $g\left(K_{2 m, 2 k+1}\right)=m$ follows. Together, these determine the knot Floer homology of $K_{2 m, 2 k+1}$ :

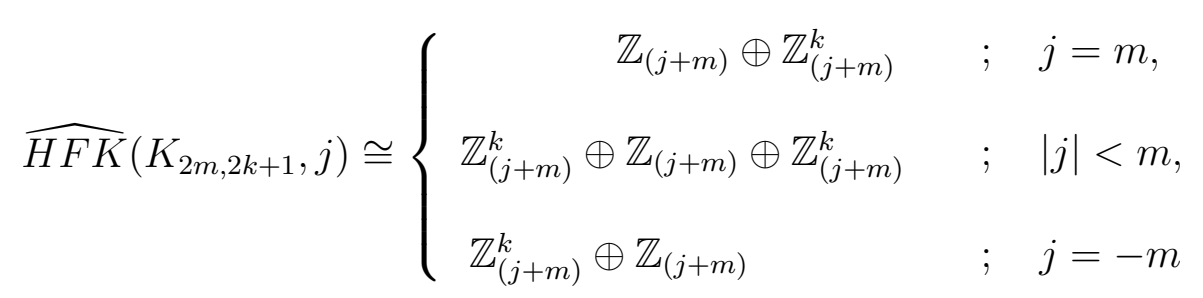

The vertical components of the $d_{2}$ differentials are then:

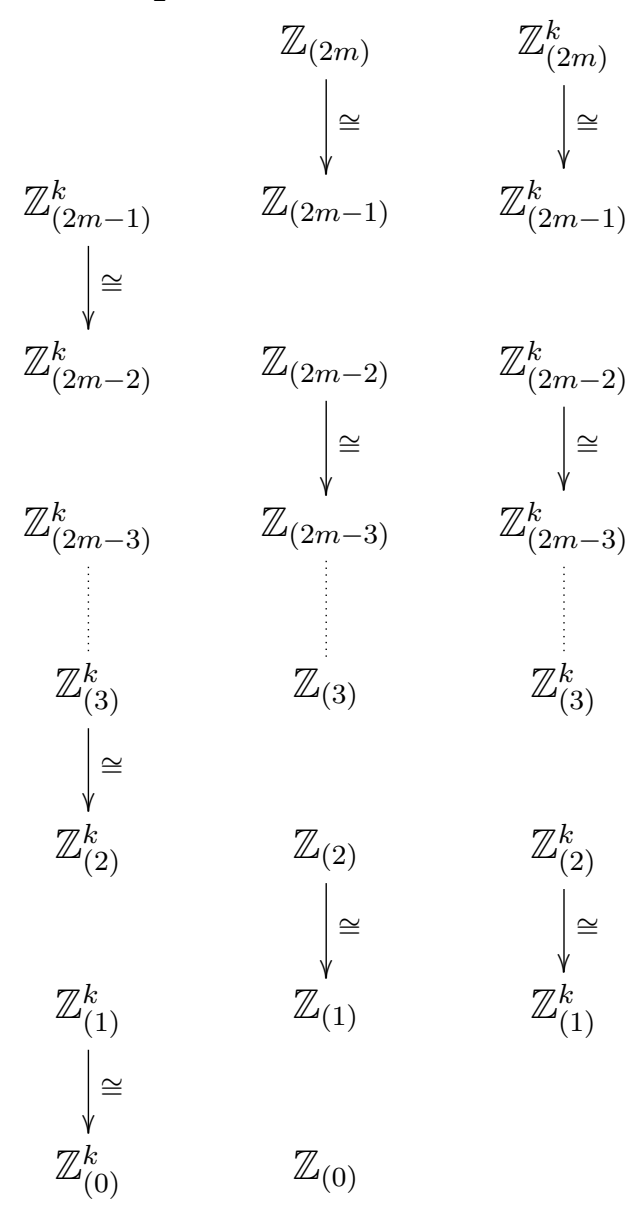

The placement of the horizontal components of the $d_{2}$ differential in relation to the vertical ones, is a slightly more delicate task. Nevertheless, this placement is uniquely determined by the underlying algebra. This is evident for the horizontal differential acting on $\widehat{H F K}\left(K_{2 m, 2 k+1},-m\right) \otimes U^{t}, t \in \mathbb{Z}$, and it is as in the large diagram on the next page. Once this differential is understood, it pins down uniquely the differential on 
$\widehat{H F K}\left(K_{2 m, 2 k+1},-m+1\right) \otimes U^{t}$. Proceeding by induction, one obtained all the horizontal $d_{2}$ differentials. This procedure leads to:

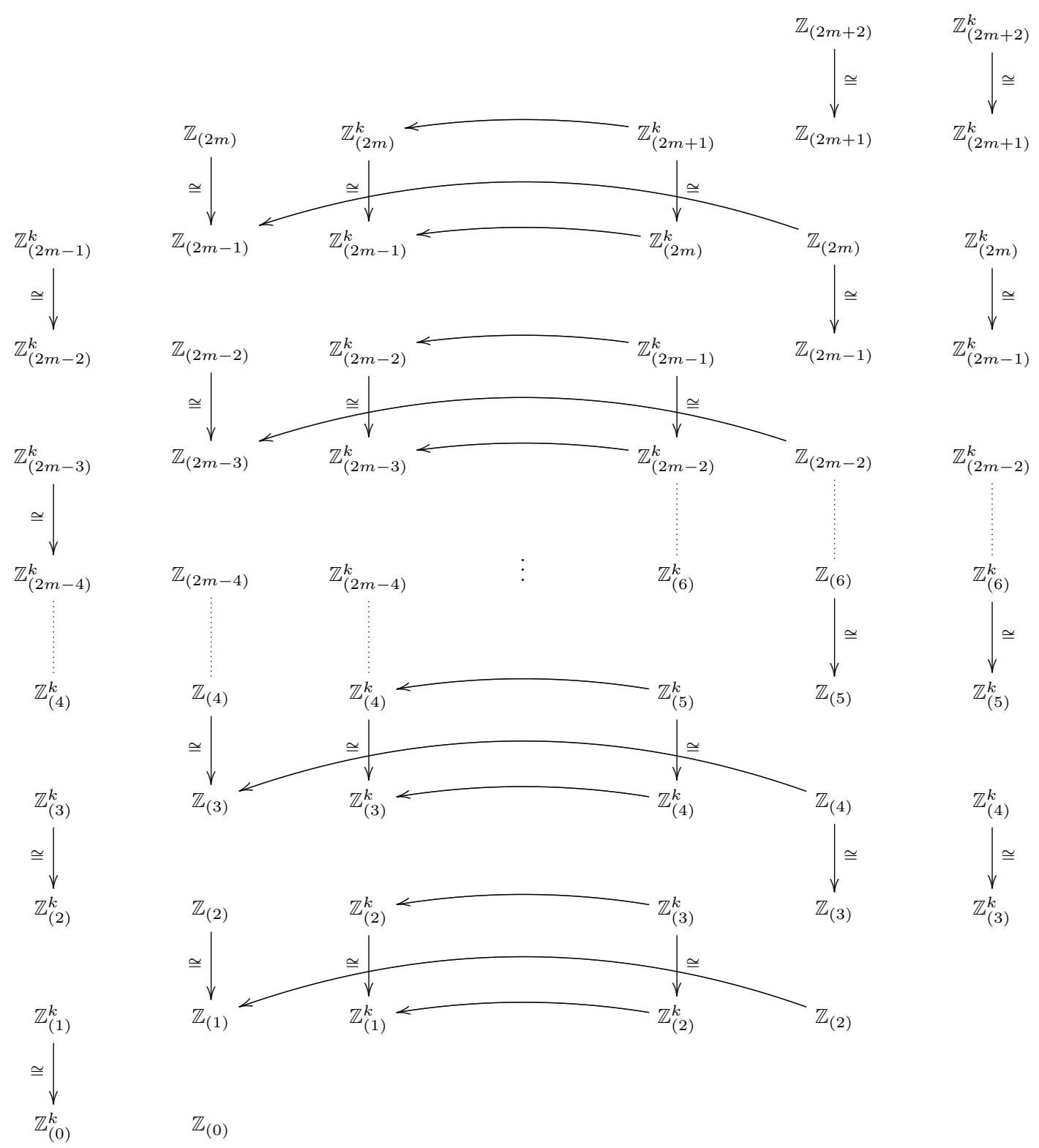

The homology of the various $\hat{A}_{s}$, is now easy to determine. Indeed, the only relevant part of $\hat{A}_{s}$ that needs examining, is the contribution from generators $[x, i, j]$ with $(i, j) \in$ $\{(0, s),(-1, s),(0, s-1)\}$. The homology depends on the parity of $s$. Firstly, if $|s|<m$ and $m-s$ is even, the relevant part looks like: 
HEEGAARD FLOER GENUS BOUNDS FOR DEHN SURGERIES ON KNOTS

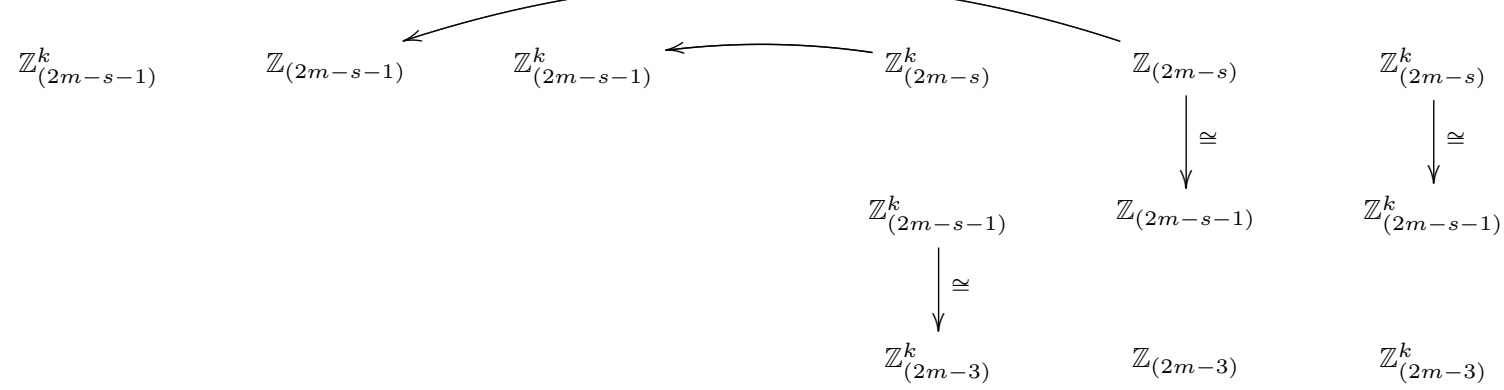

Thus, $H_{*}\left(\hat{A}_{s}\right) \cong \mathbb{Z} \oplus \mathbb{Z} \oplus \mathbb{Z}$ and $\hat{v}_{s}$ and $\hat{h}_{s}$ are the projections $\pi_{1}$ and $\pi_{2}$ onto the first and second $\mathbb{Z}$-summand respectively.

If $|s|<m$ and $m-s$ is odd, we get instead

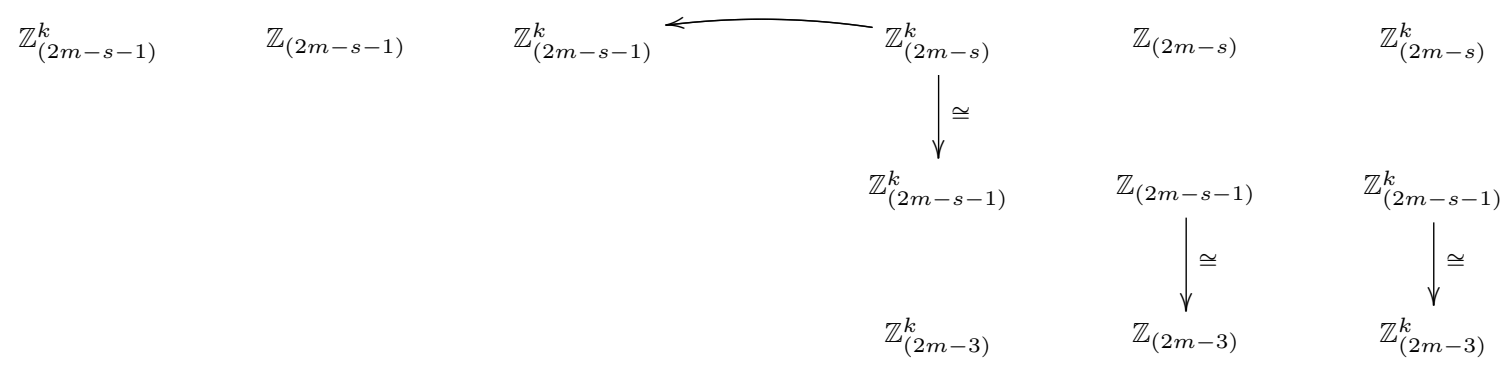

The homology of $\hat{A}_{s}$ is then isomorphic to $\mathbb{Z} \oplus \mathbb{Z} \oplus \mathbb{Z}^{2 k+1}$ and $\hat{v}_{s}$ and $\hat{h}_{s}$ are again the projections $\pi_{1}$ and $\pi_{2}$ onto the first and second $\mathbb{Z}$-summand respectively.

Assuming that $p-(2 m-1) q>0$, we proceed to compute the number of $L$-structures of $S_{-p / q}^{3}\left(K_{2 m, 2 k+1}\right)$ by dividing its $\operatorname{spin}^{c}$-structures into two distinct groups:

- Those $[i] \in \operatorname{Spin}^{c}\left(S_{-p / q}^{3}\left(K_{2 m, 2 k+1}\right)\right)$ for which $\left\lfloor\frac{i+p s}{q}\right\rfloor \leq-m$ or $\left\lfloor\frac{i+p s}{q}\right\rfloor \geq m$ for all $s \in \mathbb{Z}$.

- Those $[i] \in \operatorname{Spin}^{c}\left(S_{-p / q}^{3}\left(K_{2 m, 2 k+1}\right)\right)$ for which there exists a unique $s_{i} \in \mathbb{Z}$ with $-g<\left\lfloor\frac{i+p s_{i}}{q}\right\rfloor<g$.

Spin $^{c}$-structures of the first kind are guaranteed to be $L$-structures and there are $p-$ $(2 m-1) q$ of them. For the $\operatorname{spin}^{c}$-structures of the second kind, we need to distinguish between those $s_{i}^{\prime}=\left\lfloor\frac{i+p s_{i}}{q}\right\rfloor$ with $m-s_{i}^{\prime}$ even and odd.

If $m-s_{i}^{\prime}$ is even, they our computation of $H_{*}\left(\hat{A}_{s_{i}^{\prime}}\right), \hat{v}_{s_{i}^{\prime}}$ and $\hat{h}_{s_{i}^{\prime}}$ above show that $\widehat{H F}\left(S_{-p / q}^{3}\left(K_{2 m, 2 k+1}\right),[i]\right)$ is isomorphic to the homology of the mapping cone:

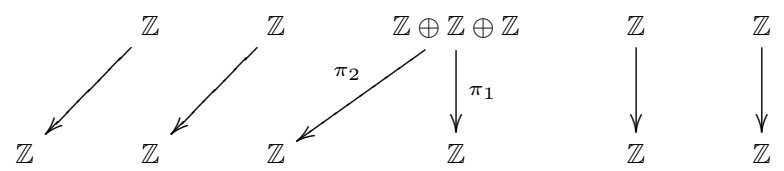

Its homology is easily found to be $\mathbb{Z}$ and so such $\operatorname{spin}^{c}$-structures are $L$-structures.

If $m-s_{i}^{\prime}$ is odd, our computations of $H_{*}\left(\hat{A}_{s_{i}^{\prime}}\right), \hat{v}_{s_{i}^{\prime}}$ and $\hat{h}_{s_{i}^{\prime}}$ show that $\widehat{H F}\left(S_{-p / q}^{3}\left(K_{2 m, 2 k+1}\right),[i]\right)$ is isomorphic to the homology of the mapping cone 


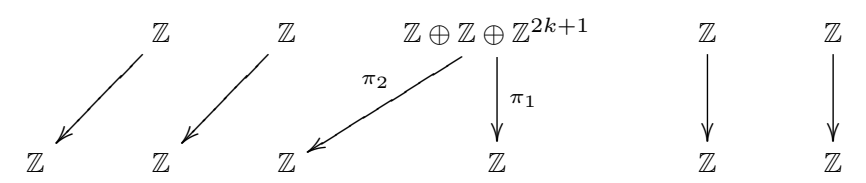

which is isomorphic to $\mathbb{Z}^{2 k+1}$. Thus, this $[i]$ is not an $L$-structure for any choice of $k \in \mathbb{N}$.

To summarize, the number of $L$-structures coming from $\operatorname{spin}^{c}$-structures of the second kind is $(m-1) q$, which when added to the number $p-(2 m-1) q$ of $L$-structures from spin $^{c}$-structures of the first kind, gives a total of $p-m q L$-structures on $S_{-p / q}^{3}\left(K_{2 m, 2 k+1}\right)$, as claimed in Example 1.10 .

To complete proving the claims made in Example 1.10, we need to demonstrate that the framed knots $\left(K_{2 m, 2 k+1},-\frac{4 m n-1}{n}\right)$ and $\left(K_{2 n, 2 k+1},-\frac{4 m n-1}{m}\right)$ are surgery equivalent. This is accomplished by a sequence of Rolfsen twists as explained in Figure 6.

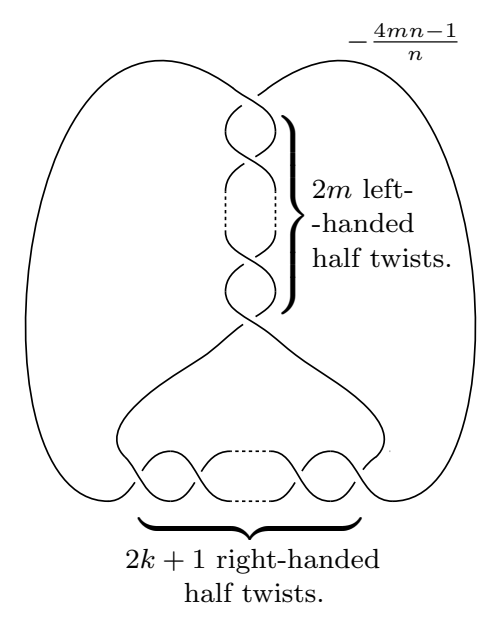

(a)

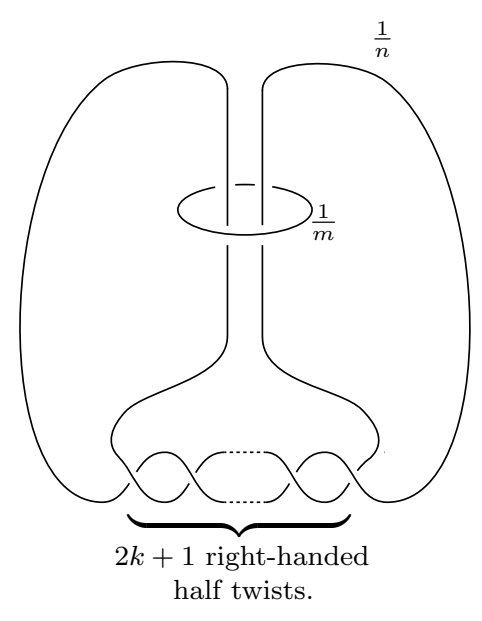

(b)

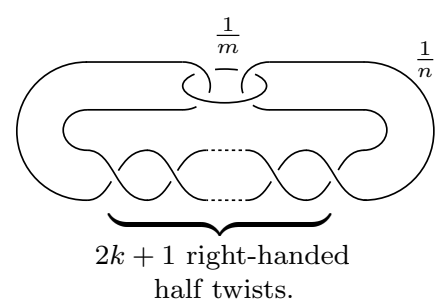

(c)

Figure 6 . The knot $K_{2 m, 2 k+1}$ with framing $-\frac{4 m n-1}{n}$ in picture (a) is obtained from the framed link in picture (b) by performing a Rolfsen twist along the unknot with framing $1 / m$. The framed link in picture (c), gotten by an isotopy from the link in picture (b), is symmetric under interchanging $m$ and $n$. Accordingly, the framed knots $\left(K_{2 m, 2 k+1},-\frac{4 m n-1}{n}\right)$ and $\left(K_{2 n, 2 k+1},-\frac{4 m n-1}{m}\right)$ are sugery equivalent.

\section{REFERENCES}

[1] W. R. Brakes. Manifolds with multiple knot-surgery descriptions. Math. Proc. Cambridge Philos. Soc., 87(3):443-448, 1980.

[2] Margaret I. Doig. Finite knot surgeries and Heegaard Floer homology. arXiv:1201.4187v1, 2012.

[3] Joshua Greene. L-space surgeries, genus bounds, and the cabling conjecture. arXiv:1009.1130v2, 2010.

[4] Akio Kawauchi. Mutative hyperbolic homology 3-spheres with the same Floer homology. Geom. Dedicata, 61(2):205-217, 1996.

[5] Robion Kirby. A calculus for framed links in $S^{3}$. Invent. Math., 45(1):35-56, 1978. 
[6] W. B. R. Lickorish. A representation of orientable combinatorial 3-manifolds. Ann. of Math. (2), 76:531-540, 1962.

[7] W. B. Raymond Lickorish. Surgery on knots. Proc. Amer. Math. Soc., 60:296-298 (1977), 1976.

[8] Charles Livingston. More 3-manifolds with multiple knot-surgery and branched-cover descriptions. Math. Proc. Cambridge Philos. Soc., 91(3):473-475, 1982.

[9] Peter Ozsváth and Zoltán Szabó. Knot Floer homology and the four-ball genus. Geom. Topol., 7:615-639, 2003.

[10] Peter Ozsváth and Zoltán Szabó. Holomorphic disks and knot invariants. Adv. Math., 186(1):58116, 2004.

[11] Peter Ozsváth and Zoltán Szabó. Holomorphic disks and three-manifold invariants: properties and applications. Ann. of Math. (2), 159(3):1159-1245, 2004.

[12] Peter Ozsváth and Zoltán Szabó. Holomorphic disks and topological invariants for closed threemanifolds. Ann. of Math. (2), 159(3):1027-1158, 2004.

[13] Peter Ozsváth and Zoltán Szabó. On knot Floer homology and lens space surgeries. Topology, 44(6):1281-1300, 2005.

[14] Peter S. Ozsváth and Zoltán Szabó. Knot Floer homology and rational surgeries. Algebr. Geom. Topol., 11(1):1-68, 2011.

[15] Jacob Rasmussen. Lens space surgeries and a conjecture of Goda and Teragaito. Geom. Topol., 8:1013-1031, 2004.

[16] Jacob Andrew Rasmussen. Floer homology and knot complements. ProQuest LLC, Ann Arbor, MI, 2003. Thesis (Ph.D.)-Harvard University.

[17] Dale Rolfsen. Knots and links. Publish or Perish Inc., Berkeley, Calif., 1976. Mathematics Lecture Series, No. 7.

[18] Dale Rolfsen. Rational surgery calculus: extension of Kirby's theorem. Pacific J. Math., 110(2):377-386, 1984.

[19] Teruhiko Soma. Simple links and tangles. Tokyo J. Math., 6(1):65-73, 1983.

[20] Masakazu Teragaito. A Seifert fibered manifold with infinitely many knot-surgery descriptions. Int. Math. Res. Not. IMRN, (9):Art. ID rnm 028, 16, 2007.

[21] Andrew H. Wallace. Modifications and cobounding manifolds. Canad. J. Math., 12:503-528, 1960.

E-mail address: jabuka@unr.edu

Department of Mathematics and Statistics, University of Nevada, Reno NV 89557. 